\title{
Il ritorno di Jiří Gruša und Beneš als Österreicher Zur Problematik des literarischen Schaffens in zwei Sprachen
}

Mojmír Jeřábek

\section{Einführung}

Es ist immer ein nicht nur literaturwissenschaftliches, sondern meistens auch ein durchaus praktisches Problem, wenn ein Autor von einer Sprache in eine andere in seinem Schaffen wechselt. Diese Erfahrung haben viele Autoren der tschechischen Literatur erlebt. Meistens war dieser Sprachwechsel Folge der politischen Ereignisse und Entwicklungen in der zweiten Hälfte des vorigen Jahrhunderts, als die Autoren, meistens im Konflikt mit dem Regime, freiwillig und mit Erleichterung, oft aber unfreiwillig das Land verlassen mussten.

In welche Literatur(geschichte) gehören diese Autoren? In die ursprüngliche? In die neue - angenommene oder gewählte? In beide? Ein ähnliches Problem wie bei Franz Kafka, wobei dieser seine (Literatur)Sprache nie gewechselt hat...

Das Problem haben wir z. B. mit Milan Kundera, den die Franzosen (mit Recht) für einen der größten zeitgenössischen französischen Schriftsteller halten - in den tschechischen Augen (und auch in seinen eigenen!) ist und bleibt er aber auch ein tschechischer Autor. Er gehört ohne Zweifel zu den Schlüsselfiguren der tschechischen Literatur: Ohne seinen Roman Žert (Der Scherz) fehlte es nicht nur an einem der Hauptwerke der tschechischen Nachkriegsliteratur, sondern es gäbe vielleicht auch nicht in diesem Ausmaß die gesellschaftliche und kulturelle Befreiungsbewegung in der ČSSR der 60. Jahre! So viel Einfluss hatte nämlich damals Literatur...

Und diesen erweiterten Raum der politischen und publizistischen Freiheit konnten dann Literaten der „neuen Welle“ perfekt ausnützen, Autoren, die als erste ohne die freiwillig angenommene oder aufgezwungene Ideologie gedacht und geschaffen haben. Leute wie Václav Havel oder Jiří Gruša, die eigentlich schon den Kern der zukünftigen demokratischen Republik gebildet haben.

Am Anfang waren Publikations- und Berufsverbote für die politischen Gegner der Kommunisten nach 1948, nach 1968 dann schon pauschal für die ganze literarische und 
geistige Elite des Landes. Meistens war es verbunden - vor allem bei den bekanntesten Namen wie eben Kundera, Havel etc. - mit einer polizeilichen Aufsicht. Die Reaktionen darauf waren schon individuell: Milan Kundera und seine Frau Věra - ohne jegliche Job-Aussicht, jedoch unter Aufsicht - haben das Angebot einer Gastprofessur in Rennes (Bretagne) für den Schriftsteller 1975 dankbar akzeptiert. Das tschechoslowakische Husák-Regime hat das für ein Jahr erlaubt, es war ihm sogar sehr recht. Vielleicht führte auch dieser Fall zu der Idee der freiwilligen und vor allem unfreiwilligen Vertreibung der Regime-Gegner ins westliche Ausland. Es bekam sogar einen zynischen Namen: „Asanace“ wohl im Sinne Sanierung oder Säuberung. Nach einem großen polizeilichen Druck auf die Autoren, bzw. regimekritische Person, hat man diesen ihre Nullzukunft geschildert oder sogar mit Gefängnis gedroht und als eine Art Lösung das freiwillige Verlassen der Heimat angeboten, ja dazu gezwungen. Von den Schriftstellern, die in diesem Beitrag erwähnt werden, wurden in Folge dieser Aktion „Asanace“ z. B. Pavel Kohout, Ota Filip oder eben Jiří Gruša betroffen. Ota Filip hat vor der Perspektive eines Gefängnisses kapituliert und ist freiwillig in die Bundesrepublik Deutschland mit der ganzen Familie übersiedelt - jedoch nicht als deutschstämmig mit dem deutschen Reisepass, sondern als offizieller Aussiedler. Pavel Kohout und seiner Frau Jelena Mašínová wurde die Rückkehr aus Österreich an der Grenze physisch verhindert, bei Jiří Gruša hat man auf die Gelegenheit zur Ausbürgerung gelauert - und mit Hilfe eines Exiltschechen erfolgreich.

Wie haben diese Autoren dann das Sprachproblem gelöst? Pavel Kohout hat seine literarische Sprache zwar nie gewechselt, jedoch das Genre. Der große und sehr fruchtbare Dramatiker wurde - aus praktischen Gründen - zum großen und sehr fruchtbaren Romancier. Milan Kundera setzte sein Romanschaffen auf Tschechisch auch im Exil fort bis 1990. Jedoch als ein Mann des Wortes par excellence, der akribisch jede Übersetzung streng mit dem Original vergleicht und beurteilt, war er immer mehr unzufrieden mit den Übersetzungen vor allem in die Sprache seiner neuen Wahlkultur und so begann er - also nicht ganz freiwillig - direkt auf Französisch zu schreiben. (Zumindest schildert er das so in seinem Werk und Erinnerungen. $)^{1}$ Ähnliches erlebte allerdings Jiří Gruša mit der ersten Romanübersetzung nach seiner Zwangsumsiedlung in die Bundesrepublik. Er war über das Missverständnis seines Romans Doktor Kokěs - Mistr Panny durch den Übersetzter, aber auch durch den Herausgeber so entsetzt, dass er letztendlich die Herausgabe nur unter einem anderen Titel - nämlich Janinka - akzeptierte). Und so

1 Dazu z. B. Milan Kundera in der Einführung zu dem Sechsten Teil Fünfundsechzig Wörter seines Essays Die Kunst des Romans: „In den Jahren 1968 und 1969 wurde Der Scherz in alle westlichen Sprachen übersetzt. Doch welche Überraschungen! In Frankreich schrieb der Übersetzer den Roman um, indem er meinen Stil ausschmückte. In England strich der Herausgeber alle Abschnitte mit Reflexionen, ließ die musikwissenschaftlichen Kapitel weg, änderte die Reihenfolge der Teile, stellte den Roman anders zusammen. In einem anderen Land. Ich treffe meinen Übersetzer: Er kann kein einziges Wort Tschechisch. „Wie haben Sie den übersetzt?“ Er antwortete: »Mit dem Herzen«, zieht ein Photo von mir aus der Brieftasche und zeigt es mir. Er war so sympathisch, daß ich fast geglaubt hätte, man könnte wirklich dank einer Telepathie des Herzens übersetzen. Ich schlage das Buch zufällig bei Helenas Monolog auf. Die langen Sätze, die bei mir jeweils einen ganzen Absatz einnehmen, sind in eine Unmenge von einfachen Sätzen unterteilt... Der durch die Übersetzungen von Der Scherz verursachte Schock hat mich für immer gezeichnet." Kundera, Milan: Die Kunst des Romans, Fischer Taschenbuch Verlag, Frankfurt am Main, 2010, S. 153 
wurde Kundera auch zu einem der größten französischen Romanciers und später auch zu einem großen Essayisten. Zu einem weltbedeutenden mitteleuropäischen Essayisten!

Wieso „mitteleuropäisch“? In Tschechien gibt es eine viel diskutierte Frage: Gehört Kundera, als französisch schreibender Essayist und Romancier, auch noch „seinen“ Tschechen und Mährern? Meine Überzeugung ist: Ganz sicher ja! Genauso wie Autoren, über die er viel geschrieben und nachgedacht hat, und die er bewundert, z. B. Franz Kafka und Robert Musil. Die Sprache ist nicht entscheidend - entscheidend sind die Themen, die Sichtweise, die Gedanken- und Kulturwelt. Und die sind bei Milan Kundera meistens nicht „französisch“, ja auch nicht „tschechisch“ im engen Sinn, sondern „mitteleuropäisch“ in der ruhmreichen Tradition. Deswegen heißt meine These: Milan Kundera ergänzt - wohl als der letzte - dieses große Gestirn der weltbekannten Mitteleuropäer, von dem er gerne und so oft mit Bewunderung schreibt!

Aber die Anderen? Ota Filip und Jiří Gruša waren zwar schon damals bekannte Namen der tschechischen Literatur, jedoch in der Welt nicht so berühmt wie Kohout oder Kundera. Umso schwieriger war es also für sie, ihre literarische Tätigkeit in der neuen Sprach- und Kulturumgebung der Bundesrepublik Deutschland fortzusetzen oder neu anzufangen. ${ }^{2}$ Filip hat sich deswegen zuerst vor allem als Journalist profiliert, obwohl „deutschstämmig“, langten seine Deutschkenntnisse nicht aus, um in dieser Sprache gleich publizieren zu können. Ähnlich jedoch wie bei Kundera, die Unzufriedenheit mit den Übersetzungen führte ihn schnell zum Sprachwechsel - und er wurde zum bekannten deutschen Journalisten und Romancier. Er bleib jedoch in seinem Schaffen zweisprachig.

Jiří Gruša war vielleicht der schwierigste Fall: unter dem großen politischen Druck als Dissident und teilweise in der daraus folgenden Isolierung verfiel er literarisch schon vor seiner Ausreise in eine „Tautologie“, wie er das z. B. in den Vorlesungen der Dresdner Poetikdozentur beschreibt. ${ }^{3}$ Nach der Ausreise wurde er durch die Ausbürgerung um alles beraubt: um seine Staatsangehörigkeit, um seine Familie, um sein Hab und Gut und vor allem um seine Sprache, die er als Mensch des Wortes par excellence unbedingt brauchte. Er musste seine Existenz vom Punkt Null neu gründen. Sein langer Weg vom tschechischen Lyriker und Romancier zum deutschsprachigen Lyriker und Essayisten viel verdankt er auf diesem Wege seiner Frau Sabine - wurde mehrmals thematisiert. ${ }^{4}$ Beschrieben wurde jedoch bis jetzt nicht genau seine Rückkehr nach der politischen Wende in die tschechische Literatur. Dieser Aufsatz bemüht sich um einen Beitrag dazu.

2 Dazu Jiří Gruša in einem (Selbst)Interview zum Thema Beneš: Literát mimo svưj jazyk, jde to? „Nejde. Nebo se musíte odhodlat ke skoku a nedivit se, když skončíte s nohou v sádře. Já jsem chtěl ale zahodit Herderův batoh, který jsme v Evropě všichni tahali. Jazyk duše národa? Ne, komunikativní know-how! A tak mě němčina nepřipravovala o češtinu, nýbrž jen o českou tíseň. Prostor jazyka byl desetkrát větší. Zmenšoval náfuky a talentům dával kyslík. (...)“. Revue Kontexty, 2/2011, S. 51

3 Gruša, Jiří: Das Gesicht - der Schriftsteller - der Fall. Literatur in Mitteleuropa. Dresdner Poetikdozentur. Thelem Universitätsverlag, Dresden 1999

4 Z.B. Cornejo, Renata: Heimat im Wort, Wien 2010 


\section{Il ritorno d'Ulisse in Patria}

Der Dramatiker Milan Uhde, Exdissident aus Brünn, machte in der Zeit, als dieser in einer der höchsten politischen Funktionen seines Landes stand, den Autor dieses Textes mit Jiří Gruša (1938-2011) bekannt. Auf Anfrage von seinem ehemaligen literarischen Kollegen und Freund aus der Charta 77 nach einem geeigneten Kandidaten für einen diplomatischen Posten in der Botschaft in Bonn wurde ich empfohlen. In der Folge durfte ich dann in den Jahren 1996-97 den Posten als Botschaftsrat und Leiter der Kultur- und Presseabteilung am Rheinufer bekleiden.

Jiří Gruša war damals für mich natürlich ein Begriff - jedoch im Rahmen der tschechischen Literatur eher historisch: aus den 60. Jahren, als er sich als Dichter, Editor und Querulant gegen den sozialistischen und stalinistischen literarischen Kanon (Verš pro kočku - Vers für die Katz) dauerhaft in die Geschichte der tschechischen Literatur eintrug. Und weil damals die Literatur in der ČSSR eine große gesellschaftliche Rolle spielte und faktisch den nicht stattfindenden politischen Diskurs ersetzte, musste der Hammer der politischen Polizei (StB) logischerweise auf den parteilosen, also nichtkommunistischen Kopf von Jiří Gruša einschlagen. Und es waren harte Schläge: wiederholte Strafverfahren, Arbeits- und Publikationsverbot. Die Folgen: Seine Arbeit im Dissens, Unterschrift der Charta 77 und dann ein erzwungenes Exil in der Bundesrepublik Deutschland, ein schweres Schicksal für einen der hervorragendsten Vertreter des unabhängigen Denkens im ganzen Ostblock. Durch den Eisernen Vorhang sickerten dann in die ČSSR - mindestens für mich - nur einige Tropfen von seiner Tätigkeit. Von seiner literarischen Tätigkeit waren mir nur seine editorischen Taten bekannt - z. B. die Anthologie der vom hiesigen Regime verbotenen Autoren. ${ }^{5}$

So entdeckte ich für mich Jiří Gruša als Autor und vor allem als Essayisten par excellence in Bonn live, bei seinen öffentlichen Auftritten als Botschafter. Ich konnte dabei vor allem seine Reden bewundern; mit Erstaunen wurde mir klar, dass jede dieser Reden ein glänzender Essay ist - eigentlich zu schade nur für diesen Moment und nur für ein kleines Publikum! Mir wurde bewusst: Hier steht vor mir wohl der bedeutendste tschechische Essayist der Gegenwart, der sich allerdings in deutscher Sprache ausdrückt. Fast alle anderen literarischen Größen in den politischen oder anderen Posten konnten keine dauerhafte und konzentrierte essayistische Tätigkeit mehr präsentieren (Havel, Pithart).

Dabei war Grušas Rolle politisch höchst schwierig: als Botschafter eines kleineren Nachbarlandes, mit dem vor allem in der Vorstellung der Vertriebenen alles offen war, und in der Heimat noch sehr stark mit der „deutschen Karte“ nicht nur von den politischen Gauklern gefuchtelt wurde (es war genau in der sehr sensiblen Zeit mitten in den Verhandlungen um die Deutsch-Tschechische Erklärung). Dieser Botschafter wurde deswegen schärfstens beobachtet und jedes sein Wort mehrmals überprüft - und das auf beiden Seiten der Grenze. Und trotzdem blieb Jiří Gruša - wie immer! - sich selbst treu und ließ sich seine Autorität, die er als Autor besaß, nie nehmen, ohne jedoch seine Heimat je im Stich zu lassen. Er konnte seine diplomatische (politische) Mission von 
der literarischen (sprachlichen) nie voll trennen. Seine literarischen und essayistischen Fähigkeiten, seine einzigartigen Sprachkompetenzen in beiden Sprachen trugen wesentlich zum Erfolg der Deutsch-Tschechischen Erklärung bei.

Ich konnte also täglich erleben, wie der Botschafter und Autor in einer Person mit seinen Reden die Aufmerksamkeit, den Respekt und nicht zuletzt die Herzen seiner Zuhörer gewann. Diese hohe Anerkennung und Autorität, die er bei der deutschen Öffentlichkeit und Politik errang, hat er dann auch in Österreich, als er in den Jahren 1998-2004 tschechischer Botschafter in Wien wurde, mit denselben Mitteln gewonnen: d. h. mit seinem Charisma, seiner Offenheit, seiner Intelligenz - und seinen offiziellen Auftritten und Reden. Sein Stern glänzte bald auch bei unserem südlichen Nachbarn genauso wie nach wie vor in seiner zuerst erzwungenen, dann aber geschätzten neuen Heimat - Deutschland.

Und wer war er in seiner ursprünglichen Heimat? Was für ein Kontrast! Bei den politischen und kulturellen Spitzen in den deutschsprachigen Ländern bekannt, auch bei seinen Gegnern respektiert und von vielen geschätzt und bewundert, mit höchsten politischen Orden und literarischen und künstlerischen Auszeichnungen gewürdigt. In der Tschechischen Republik war dagegen Gruša viel weniger bekannt: vielleicht nur als unser Botschafter und aus den Medien als ein höchst intelligenter Mann und gefragter Interviewpartner - aber nicht als aktueller Buchautor! Auf dem unübersichtlichen Buchmarkt nach dem Fall der ideologischen Barrieren nach 1989 erschien nämlich nur ein Titel von Jiř́ Gruša, und zwar Dotaznik - Der 16. Fragebogen, eigentlich eine Neuauflage seines Romans, der ursprünglich im tschechischen Exilverlag Sixty-Eight Publishers bei dem Ehepaar Škvorecký in Toronto herausgebracht wurde. In der Flut der nach zwanzig oder sogar vierzig, ja fünfzig Jahren stürmisch nachgeholten Titel ist dieses Buch untergetaucht - wohl nicht ganz zum Bedauern des Autors, wie er mehrmals mit einem Wermuttropfen erwähnte. Er hat nämlich noch kurz vor der Wende gemeinsam mit Tomáš Kosta in Köln und den tschechischen Dissidenten aus Brünn die Entstehung eines vom Regime unabhängigen Verlags in die Wege geleitet (der Clou steckte in der Form - einer Genossenschaft, die die Genossen gerade wohlwollend erlaubt haben - und dabei nicht an diese Dreistigkeit gedacht haben - gedacht war vielleicht an kleine Handwerker, aber nicht an die „Wordseller“!) So entstand in Brünn unter dem direkten Einfluss von Jiř́ Gruša der Atlantis-Verlag, der dann den Dotaznik herausgebracht hat - laut Jiří Gruša sogar mit der entscheidenden finanziellen Unterstützung des Autors, der jedoch mit dem Ergebnis sehr unzufrieden war. Und sonst fast nichts: der Lyriker Jiří Gruša war in einer winzigen Auflage präsent, wenn man das so überhaupt behaupten könnte. Ein Lyrikband Modlitba k Janince, seine Gedichte aus den Jahren 1969-1973 erschien zwar im großen Verlag Český spisovatel in Prag 1994 - jedoch nur als eine Bibliophilie in 100 Stück; das war wohl nicht viel mehr als der Samisdat 1975... Außerdem gab es bis zu dem weiter geschilderten essayistischen Durchbruch 2001 mit dem Band Česko-Návod $k$ použití nur noch einen Titel mit seinem Namen, und zwar seine deutsche Lyrik aus den Gedichtbänden Babylonwald und Wandersteine in der Übersetzung seines Arbeits- und Dichterkollegen aus dem Prager Außenministerium Tomáš Kafka - mit der Übersetzung war der Autor allerdings nicht ganz zufrieden. ${ }^{6}$

6 Gruša, Jiř̌i: Les Babylon, Bludné kameny, Torst, 1998 
Also ein Paradox: Ein anerkannter deutscher Buchautor, Editor, Essayist und Dichter - siehe die Publikation über die 1000 Jahre deutscher Lyrik, ein Kanon, in dem Jiř́i Gruša nicht fehlen durfte ${ }^{7}$ - der jedoch kein deutscher Staatsbürger mehr war, und ein fast unbekannter Autor seines Landes, das er jetzt - wieder mit dem tschechischen Pass in der Tasche - vertrat!

Nach meiner Rückkehr aus der diplomatischen Mission in Bonn blieben wir mit Jiří Gruša in Kontakt. Nach seinem großen literarischen Erfolg mit dem Bestseller Gebrauchsanweisung für Tschechien (später in weiteren Ausgaben ergänzt noch um die Erweiterung „und Prag“) wunderte ich mich erneut, warum er in der Tschechischen Republik nicht auf dem Buchmarkt präsent sei, und fragte ihn danach. Seine Antwort war: Es gibt keinen Verlag, der sich für meine Bücher interessieren würde. Danach bot ich ihm die Gelegenheit, auch in seiner Heimat auf den Markt mit seinen Büchern zurück zu kommen und zwar nach Absprache mit den Managern und Inhabern des Brünner Verlags Barrister \& Principal František Mikš und Ivo Lukáš. Sie zeigten großes Interesse für die tschechische Fassung von Gebrauchsanweisung.

Diese Zusammenarbeit dauert bis heute: Seit 2014 erscheint in diesem Verlag die zehnbändige Werkausgabe von Jiří Gruša.

Einen tschechischen Text aus der Gebrauchsanweisung vorzubereiten war jedoch keine leichte Aufgabe. Der Text - im Auftrag geschrieben für den Münchner Piper-Verlag - war für den deutschen Leser bestimmt und voll von vielen für den Autor typischen Wortspielen, Alliterationen etc. Das Buch entstand binnen sehr kurzer Zeit von etwa drei Monaten im Jahre 1998 in einer Lücke, als Jiří Gruša keine Beschäftigung ausübte nach dem Fall der tschechischen Regierung, in der Jiří Gruša vom Ministerpräsidenten Václav Klaus in den Posten des Schulministers berufen wurde.

Der Autor war jetzt zwar über die neue Publikationsmöglichkeit, die er als eine Art Satisfaktion verstand, sehr erfreut, jedoch inzwischen wieder mit seinem neuen Job als tschechischer Botschafter in Wien voll beschäftigt. Und dieser hohe Posten in Wien zeigte sich viel schwieriger als vom Botschafter erwartet: gleich nach seinem Antritt kamen nämlich in Österreich mit voller Wucht Protest-Themen wie Atomkraftwerk Temelín oder die Beneš-Dekrete auf. Das waren keine guten Voraussetzungen für eine systematische literarische Arbeit! Um dem Autor nicht davon abzuraten und ihn wirklich für den tschechischen Leser (und den Verlag) zu gewinnen, bot ich ihm an, dass ich für ihn Stück für Stück eine Arbeitsfassung der Übersetzung vorbereite, mit der er dann weiterarbeiten könnte. Der Autor akzeptierte dieses Verfahren und aus dieser Praxis entwickelte sich eine jahrelange und erfolgreiche Zusammenarbeit. Diese bedeutete für mich eine große Bereicherung, weil es sich auch um viele Stunden handelte, die wir in gemeinsamer Arbeit an den Texten verbrachten. Ich konnte bei den Konsultationen und Neuformulierungen von vielen Textstellen seine unvergleichbare Denkweise kennenlernen und die einzigartige Formulierungskunst des Autors im Entstehen erleben. Der Autor war natürlich derjenige, der aus den Arbeitsübersetzungen diese hervorragenden

$7 \quad$ Das große deutsche Gedichtbuch von den Anfängen bis zur Gegenwart. Neu herausgegeben und aktualisiert von Karl Otto Conrady. Düsseldorf, Zürich: Artemis und Winkle, 2000 
literarischen Texte produziert hat! Dank seiner Freundlichkeit stehen jedoch in allen Bänden die beiden Namen der Übersetzer (also Gruša, Jeřábek) nebenaneinander.

Und so begann „Il ritorno d'Ulisse in Patria“ und zwar so erfolgreich, dass man nur staunen konnte. Die Gebrauchsanweisung, jetzt als Česko - Návod k použití, wurde unerwartet zum Bestseller. Auf dem tschechischen Markt erreichte der Titel in zwei Auflagen (die zweite war eine erweiterte) etwa 5.000 verkaufte Stücke, was im theoretischen Vergleich so viel wie 50.000 Stück auf dem deutschsprachigen Markt bedeuten würde!

Es gab natürlich einen Grund für diesen Großerfolg: Im Vergleich zu den Romanen von Jiří Gruša war dieses kluge Buch einfacher zu lesen und ein echter Lesegenuss! Dabei erweist die Gebrauchsanweisung alle intellektuellen Qualitäten des Autors, sogar konzentriert. Als ein essayistisch-belletristisches Meisterwerk präsentiert es sich mit allen typischen Merkmalen seines Autors: die tschechischen Themen und Narrative neu historisch und philosophisch überdacht und interpretiert - das Ganze aus dem sehr hilfreichen Ein-Tausend-Kilometer-Abstand betrachtet. Und es wurde von vielen Lesern akzeptiert, dass diese gelehrte Sichtweise, die philosophische und historische Bildung des Autors und der notwendige Abstand vom „tschechischen Teich“, teilweise zum „Ikonoklasmus“ im „tschechischen Himmel“8 führten. (Diese „Bilderstürmerei“ fand dann ihren Höhepunkt in Beneš als Österreicher.) Es gab nur vereinzelte Gegenstimmen, die überwiegende Rezeption des Werkes war positiv. Nicht nur für die intellektuelle Öffentlichkeit bedeutete die Gebrauchswanweisung eine Entdeckung: einen wirklich neuen und überraschenden Autor.

Nach der Gebrauchsanweisung folgte im Rahmen unserer Zusammenarbeit ein anderer essayistischer Band: Štastný bezdomovec. Bei diesem Buch ging es nicht nur um die Übertragung ins Tschechische, sondern auch um eine neue Komposition des Bandes, der aus zwei verschiedenen deutschen Titeln zusammengestellt wurde. Aus dem Original - aus Glücklich heimatlos - wählte der Autor nämlich nicht alle Texte, ${ }^{9}$ und mit der bekanntlich etwa um ein Drittel kürzeren tschechischen Fassungen derselben deutschen Texte, war es wünschenswert, die tschechische Edition um weitere Texte zu ergänzen. Wir haben uns für die wirklich existenziellen Texte der Dresdner Vorlesungen entschieden. Die sogenannte Poetik-Dozentur wurde von der Technischen Universität Dresden organisiert. Jeder eingeladene Autor konnte in einer kleinen Vortragsreihe seine eigene Poetik erklären und näherbringen (von den Autoren, die uns in diesem Zusammenhang interessieren, wurde z.B. Ota Filip dazu eingeladen). Danach erschienen die Vorträge in Buchform in dem Universitätsverlag Thelem. Das war auch der Fall bei Jiří Gruša, und so entstand das schmale Büchlein mit dem Titel der drei Vorträge Das Gesicht - der

8 „Der tschechische Himmel“ - „České nebe“ - kommt in einem Kapitel vor (Gruša, Jiří: Gebrauchsanweisung für Tschechien und Prag, Piper München Zürich, 2003) mit der Reflexion, mit wem dieser Himmel besetzt wird. Dieses Motiv wurde dann ohne Erlaubnis des Autors von der populären Prager Bühne Divadlo Járy Cimrmana übernommen und unter dem Titel České nebe aufs Repertoir gesetzt. Jiří Gruša sprach in diesem Zusammenhang im Scherz davon, dass es schade ist, dass er kein Copyright auf seine Ideen anwendet - es sei nicht zum ersten Mal, dass jemand seine Idee entwende, hat er dazu gemeint.

9 Vgl. mit Gruša, Jiř́i: Glücklich heimatlos, Hohenheim Verlag, Stuttgart - Leipzig 2002 
Schriftsteller - der Fall. ${ }^{10}$ Auf dem Umschlag findet der Leser überraschende und nicht leicht zu entziffernde Zeichnungen: Hinweise zu der faszinierendsten Story aus dem Anfang des Exillebens des Schriftstellers. Der Fall bedeutete nämlich nicht nur seinen Fall auf den absoluten physischen und psychischen Boden, sondern auch, dass er zu einem interessanten Fall in der Medizin wurde, und in der Bonner Universitätsklinik auf dem Venusberg den Medizinstudenten als Objekt vorgeführt wurde. Er, der immer ein Subjekt war! Ein lyrisches, episches, dramatisches, schriftstellerisches, menschliches - Subjekt... Auch die anderen Vorträge waren Schlüsselgeschichten aus dem Leben des Autors in essayistischer Form: Tvár - Das Gesicht also war nicht nur der Titel der legendären ersten nicht-ideologischen literarischen Zeitschrift in der ČSSR, zu deren Gründern Jiří Gruša gehörte, sondern auch der Titel der Akte der politischen Polizei geführt gegen den jungen Dichter. Diese Akte wurde später geschlossen - das Objekt, eigentlich „der“11, wurde jedoch später wieder durch seine Tätigkeit in den siebziger Jahren (vor allem als Organisator des unabhängigen literarischen Betriebes und seiner eigenen literarischen Texte) zum Titel einer neuangelegten Akte: diesmal war er schon Spisovatel - Der Schriftsteller.

Česko - Návod $k$ použití machte nicht nur die Leser auf den Autor aufmerksam, sondern auch die Verleger: plötzlich tauchte im Großverlag Mladá fronta in Prag eine Neuausgabe von Dotaznik auf - diesmal schon zur vollen Zufriedenheit des Autors. Der renommierte Brünner Verleger und Autor Martin Reiner (früher Martin Pluháček) bot Jiří Gruša an, in seinem Verlag Druhé město ${ }^{12}$ die erste offizielle tschechische Ausgabe des Romans Dámský gambit (Brünn 2010) - Damengambit (Il ritorno d'Ulisse in Patria) zu publizieren.

Grušas il ritorno in Patria wurde vollbracht!

Nach dem Durchbruch im Barrister-Verlag mit Česko gab es noch in demselben Jahr 2001 einen neuen Band der original tschechischen Lyrik Grušas Wacht am Rhein aneb Putowni ghetto. ${ }^{13}$ Wieder keine neuen Gedichte, jedoch seine tschechischen Texte aus den Jahren 1973-1989 - die zeitliche Fortsetzung nach Janinka. Es war also bis zu dem Dato eine komplette bis jetzt unveröffentlichte Retrospektive, weil in der Zeit nach 1989 Gruša fast keine tschechische Lyrik mehr schrieb. Der Rückblick auf das dichterische Schaffen Grušas aus der Vorexilzeit wurde mit einem Geburtstagsband vollendet: 2003 erschien zum 65. Geburtstag des Autors im Akropolis-Verlag in Prag Právo útrpné. ${ }^{14}$ Unter dem Titel des bisher nicht veröffentlichten Gedichtbandes ging es um die Neuausgabe seiner

10 Gruša, Jiří: Das Gesicht - der Schriftsteller - der Fall. Literatur in Mitteleuropa. Dresdner Poetikdozentur. Thelem Universitätsverlag, Dresden 1999.

11 In der StB-Sprache war das Objekt so dekliniert, als ob es um „den“ Objekt ginge (z. B. „sledování objekta" etc.).

12 Ein kleiner exklusiver Verlag, wo Autoren wie Michal Viewegh, der meist verkaufte tschechische Autor mit mehr als einer Million verkauften Büchern, oder Jiří Kratochvil, der auch international angesehenste tschechische Nicht-Exil-Autor, erscheinen.

13 Gruša, Jiří: Wacht am Rhein aneb Putovni ghetto, Paseka, Praha - Litomyšl 2001.

14 Diese Ausgabe war auch eine Art „Samisdat“ - mit finanzieller Unterstützung von Nadace Charty 77 und 60 Freunden und Bekannten von Jiří Gruša - u. a. Václav Havel, Pavel Kohout, Petr Pithart, Milan Uhde, Helmut Zilk. 
kompletten Gedichtbände aus den sechziger Jahren, erweitert um den schon erwähnten nicht veröffentlichten Band, den der Autor 1964 nach den massiven Attacken gegen seine Person und Strafverfolgung - alles in der Folge seiner literarischen Tätigkeit in der Zeitschrift Tvářr - vom Verlag Mladá fronta selber zurückzog.

Nach der zweiten teilweise überarbeiteten und erweiterten Auflage von $\check{C}_{\text {esko }}{ }^{15}$ setzte unsere Zusammenarbeit kurz vor dem viel zu frühen und unerwarteten Ableben des Autors fort - mit einem kleinen Erzählungsband. Drei Texte, zwei schon vor Jahren in den schwer zugänglichen Exilpublikationen erschienen, der dritte Text, der den Titel des Bandes lieferte - Život v pravdě aneb Lhani z lásky ${ }^{16}$ war völlig neugeschrieben. Der Autor hatte ihn lange im Kopf getragen, jedoch aus ethischen Gründen zögerte er, ihn zu Papier zu bringen, bis die Hauptfigur dieser Liebesgeschichte aus seiner Jugend nicht mehr unter den Lebenden war. Inzwischen erschien auch ein langes Interview mit Dalibor Dobiáš in der Buchform Uměni stárnout, ${ }^{17}$ die Lyrik von Jiří Gruša erntete hohe Literaturpreise (Magnesia Litera, Jaroslav-Seifert-Preis), ${ }^{18}$ Česko wurde für Magnesia Litera in der Kategorie Übersetzungen nominiert etc. Der triumphale Rückzug in die tschechische Literatur wurde auch offiziell gekrönt.

Nachdem sich 2009 der Autor aus allen öffentlichen Funktionen in sein Merler Refugium zurückzog, blieb ihm ein einziges, aber für ihn persönlich besonders wichtiges Thema zu bezwingen, ein Thema, das ihn jahrelang begleitete und beschäftigte: Edvard Beneš. $^{19}$

15 Gruša, Jiří: Česko - návod k použití, Barrister \&Principal, Brno 2009 mit dem Hinweis auf dem Umschlag: Neue, erweiterte und überarbeitete Ausgabe eines erfolgreichen Buches. Diese Ausgabe beruht auf der dritten deutschen Ausgabe. Einige Kapitel wurden nach dieser Ausgabe überarbeitet, es kam ein neues Kapitel dazu: „Moravské pole aneb Jak nám zabili Otakara.“ Mehr dazu der Autor in der Bemerkung, S. 190.

16 Auf Deutsch: Das Leben in der Wahrheit oder Das Lügen aus der Liebe.

17 Gruša, Jiř́i: Uměni stárnout (Rozhovor s Daliborem Dobiášem), Paseka, Praha a Litomyšl 2004. (Der Titel auf Deutsch: Die Kunst zu altern).

18 Magnesia Litera und Cena Jaroslava Seiferta, beide 2002, beide für Jiří Gruša: Wacht am Rhein aneb Putovni ghetto

19 Dazu Jiří Gruša in dem oben zitierten Interview, S. 45 (Anm. 2): ... Proč tedy právě Beneš? Čím může historika a literáta ještě zaujmout? „On se mi tak trochu pomstil. Zabýval jsem se Masarykem, filozoficky a historicky, Beneš mě spíš zlobil. Jeho druhou kapitulaci jsem zažil jako desetiletý kluk s následky pro naši rodinu. Táta musel do dolů a uškliboval se, kdykoli padlo jeho jméno. Za mnichovské krize sloužil v pohraniční pevnůstce a nelíbilo se mu, že jsme se vzdali. Mohl se ale vrátit k rodině, kde jsem byl čerstvý kňoural. V osmašedesátém jsem si pak kladl otcovu otázku: jak to, že se nebráníme? Brzy na to jsem se setkal s Prokopem Drtinou, čímž začalo moje benešování. Zarazila mě jeho nevraživost k někdejšímu nadřízenému. Zatímco pro předválečného prezidenta hledal omluvné argumenty, únorový mu vadil. Znamenal mu konec demokracie, zaviněný nedostatkem kuráže. Udivila mne ale především jedna věta. Drtina tvrdil, že ho Beneš varoval před vstupem do politiky. »Tam Vám nebudu moci pomoct! « A taky nepomohl... „Drtina se na protest proti puči pokusil o demonstrativní sebevraždu a byl zatčen jako provokatér. Paní Drtinová prosila Beneše, který byl ještě v úřadě, o zákrok. Ten ale nehnul prstem. Z toho jsem vyvodil dvojí morálku. Politika a etika nesouvisely. Beneš se choval jako chytrý starosta, ne jako moudré kníže. To mi potvrdil i Václav Černý při našich setkáních ve Slavii. Kdykoli padlo prezidentovo jméno, mávl rukou anebo vyprávěl, jak se s ním sešel jako s depresivním mužíčkem, který všecko svádí na jiné. (...).“ 


\section{Beneš jako Rakušan im Vergleich zu Beneš als Österreicher Entstehungsgeschichte und Textvergleich}

Ich wusste schon aus meiner Bonner Zeit, dass sich der Autor als gelehrter Historiker und Philosoph und professioneller Diplomat mit dem Thema Beneš intensiv beschäftigt: Damals hat sein Studienkollege, Historiker und Beneš-Spezialist, Milan Hauner, ${ }^{20}$ auf seine Einladung einen Vortrag in der Bonner Botschaft zu diesem Thema gehalten und es gab danach eine intensive Debatte zwischen den beiden Historikern (Gruša - Hauner) auch u.a. über die Beneš-Literatur.

Trotzdem hätte es passieren können, dass das „Psychogramm“, wie es der Autor bezeichnet, Beně jako Rakušan (Barrister \& Principal, Brünn 2011) und dann seine nachfolgend), Academiae deutsche Fassung Beně̌ als Österreicher nicht entstanden wären, wenn ich dem Autor die Publikationsgelegenheit nicht angeboten hätte. Das war wohl der definitive Impuls dazu, dass sich der Autor ans Werk begab, um aus dem deutschen Manuskript ein völlig neues Buch für den tschechischen Leser zu verfassen. Im Hinblick auf unsere bisherige Zusammenarbeit und vor allem um ihn definitiv für die Arbeit zu gewinnen, habe ich Jiří Gruša wieder angeboten, dass ich das Manuskript von etwa 70 Seiten für ihn übersetze. Der Grund war nicht nur dem Autor Zeit zu verschaffen, sondern auch ein psychologischer: Dem Autor war bewusst, wenn er selber die Übersetzung seines eigenen Textes in Angriff nimmt, dass er so ein neues Buch zu schreiben beginnt - bedingt durch Umformulierungen, Verbesserungen und Textänderungen. Das ist ihm dann wirklich bei der späteren Übertragung ins Deutsche teilweise und in Details passiert. Dazu kommen wir in weiteren Ausführungen noch zurück.

Unsere Zusammenarbeit mit dem entstehenden tschechischen Text war diesmal noch grundlegender und gründlicher als je zuvor. Dem Autor lag eine rein „technisch“ korrekte Übersetzung als Grundlage für seine Arbeit vor und er konnte sich voll seinen sprachlichen Finessen und dem stilistischen „Schleifen“ des Textes widmen. Gleichzeitig jedoch im Gespräch mit mir über diejenigen Textstellen, die mir nicht klar waren, wurde ihm bewusst, dass einige seine Formulierungen viel zu verkürzt ausgedrückt sind, man muss sie doch manchmal etwas breiter erklären, ergänzen etc. Diese für den Autor typische knapp gefasste Ausdruckweise ging einerseits aus seiner breiten Bildung hervor (die Dinge waren ihm eben klar, weil er eine Menge von Fakten und daraus folgenden Kontexten wusste), andererseits jedoch auch aus seinem dichterischen Habitus. Unter diesem Einfluss hat der Autor viele Stellen zum besseren Verständnis umgearbeitet.

Ein spezielles Problem stellte das Lösen der Sprachspielereien (Alliterationen etc.) dar. Es ist uns jedoch gemeinsam gelungen, diese beinahe perfekt aus einem Sprachmilieu in das andere zu übersetzen. Wie ist das gelaufen? Ich habe mich immer zuerst bemüht eine sprachliche Lösung zu finden und diese dem Autor anzubieten. Er hat es entweder akzeptiert oder eine andere Lösung gefunden oder die Textstelle geändert, wenn

20 Českoamerický profesor Milan Hauner je specialista na středoevropské moderní dějiny, připravil mj. k vydání Paméti Edvarda Beneše (1938-1945), Praha 2007. 
keine gute Lösung gefunden werden konnte. Die für mich unvergesslichsten Momente unserer Zusammenarbeit mit den Texten waren eben die Situationen, wo wir an eine Textstelle gestoßen sind, für die ich auch bei höchster Anstrengung keine entsprechende Lösung in tschechischer Sprache finden konnte. Ich habe den Autor darauf aufmerksam gemacht - und konnte ihn direkt bei seinem literarischen Schaffen beobachten - ich war drinnen im Entstehungsprozess eines literarischen Werkes von Jiří Gruša!

An dieser Stelle muss eine wichtige Bemerkung gemacht werden: Was in dem essayistischen Werk von Jiř́i Gruša als ein Geysir von augenblicklichen Ideen des Autors erscheint, war in Wirklichkeit ein Ergebnis von tagelanger Gedankenarbeit und Reflexionen. Das bezeugen Grušas Tage- und Notizenbücher, die in seinem Nachlass bewahrt werden. Jiří Gruša hatte immer ein kleines Notizbuch dabei, wo er sich seine Ideen meistens tagsüber immer vermerkt hat, um am Abend an diese zurückzukommen. Dann hat er weiter diese Ideen durchdacht, darüber gegrübelt, ziseliert etc. Und dann setzte er diese bereits vorbereitete und überraschend formulierte oder weitsichtige Idee, Aussage oder Spruch wie einen Mosaikstein in einen passenden Kontext seiner Rede, seines Buches oder Essays, wo er plötzlich wie ein glimmender Diamant wirkt; der Leser oder Zuhörer ist der Meinung, eine plötzliche geniale Idee des Autors, die wie ein Blitz aus dem intellektuellen Himmel kommt, beizuwohnen...

Das Resultat dieser gemeinsamen Arbeit war im Vergleich zu der von mir vorbereiteten Arbeitsunterlage ein teilweise neuer oder innovierter Text und vor allem ein Text, den der Autor als definitiv und autorisiert oder kanonisiert für jede weitere Verwendung bezeichnet hat und so kodifiziert. Das ist wichtig zu betonen, um Spekulationen wie die von Aleš Knapp zurückzuweisen und ihnen zu widersprechen. ${ }^{21}$

Das oben gesagte betrifft jedoch nicht nur die Textform, sondern auch die inhaltliche und faktische Fehlerlosigkeit. Weil es sich in diesem Falle nicht um Belletristik, sondern um ein Essay handelte, das jedoch auf historischen Fakten basierte, mit vielen Hinweisen zu Sekundärliteratur und mit umfangreichem Anmerkungsapparat versehen - also aus dieser Hinsicht ging es um eine Fachliteratur - musste alles sehr akribisch überprüft und wenn nötig ergänzt und korrigiert werden. Das war wieder meine Aufgabe. Ich habe alle Fakten, inwiefern das möglich war, überprüft, den Autor auf eventuelle Fehler, Gegensätzlichkeiten, Unstimmigkeiten oder Unklarheiten aufmerksam gemacht und ihn gebeten, seine Quellen noch einmal zu überprüfen. Es ist nämlich wichtig zu sagen, dass der Autor in der Vorbereitungsphase und beim Recherchieren zum Thema Beneš eine Unmenge von Fach-, Primär- und Sekundärliteratur durchstudiert hat. Eine genauere Vorstellung davon kann man sich im Jiří-Gruša-Archiv machen, das sich im Mährischen Landesmuseum (Moravské zemské muzeum) in Brünn befindet. Wenn man heute das nicht sehr umfangreiche Buch in der Hand hält (der reine Textteil in $\mathrm{BJR}^{22}$ umfasst nur

21 Aleš Knapp, in Deutschland lebender tschechischer Publizist und Literaturkritiker, hat einen Artikel zum Vergleich der beiden Fassungen von Grušas Beneš geschrieben und den literarischen Revuen Host und Tvar angeboten. In diesem Text behauptete er, dass die tschechische Fassung später entstanden ist und vom Verlag zensuriert wurde!

22 Hier und im weiteren Text die Verkürzungen für die Titel der beiden Ausgaben:„Beneš jako Rakušan“ BJR und „Beneš als Österreicher“ - BAÖ 
knappe hundert Seiten, in BAÖ nicht einmal hundert fünfzig!), muss man zitieren, was Jiří Gruša dazu oft gemeint hat: „Es ist kein Problem, ein Fachbuch über Beneš mit tausend Seiten zu schreiben, jedoch dasselbe auf hundert Seiten zu formulieren, ist schon viel schwieriger!“

Den tschechischen Text für den Brünner Verlag beendete der Autor im Spätherbst des Jahres 2010. ${ }^{23}$ Er hat ihn als definitiv bezeichnet. Im Sommer 2011, wie schon gesagt, hat er nach diesem tschechischen Text laut seiner Aussage die deutsche Fassung überarbeitet und ergänzt.

Er selbst erläutert die Entstehungsgeschichte in beiden sprachlichen Ausgaben in der kurzen „Einleitung/Úvodem“ folgendermaßen (die Textänderungen oder Abweichungen gegenüber dem originalen tschechischen Text sind mit Kursivschrift gekennzeichnet):

„Das ganze begann mit einer Vortragsserie, oder noch besser - mit Lesungen (Bonn, Berlin, Wien, München), die heute die Grundlage des ersten Teiles des Buchs bilden. Mit der Zeit wuchsen auch die Nuancen. Im Jahre 1999 konnte ich an der Wiener Universität ein Symposion mitgestalten, das später dank Professor Arnold Suppan und Elisabeth Vyslonzil in einem Sammelband seine Endform fand: »Edvard Beneš und die tschechoslowakische Außenpolitik 1918-1948 (die zweite Auflage 2003). Zu derselben Zeit (2002) erschien in der tschechischen Botschaft in Wien unter dem Titel »Vertriebene Geschichte eine trockene Dokumentation der deutsch-nationalen Argumente gegen die Tschechen, wissenschaftlich bearbeitet von meinem Vertreter, Botschaftsrat Dr. Jiří Beránek.

Bald zeigte sich jedoch, dass ich auch über die tschechischen Anomalien sprechen musste. Und so hat sich Beneš für mich zum wiederholten, nicht immer amüsanten Thema verwandelt. Dennoch geht es in diesem Buch um keine Biografie, ich war mehr oder weniger bemüht, ein Psychogramm zu liefern, selbst wenn darin neue und überraschende Details nicht fehlen, wie Hitlers tschechische Konnotationen in Wien (in der Beilage von Hannes Brauner). ${ }^{24}$ Ich wollte und konnte nicht mit den ausgezeichneten Arbeiten ad personam Eduardi wetteifern (wie B. Čelakovský, ${ }^{25}$ J. Dejmek, K. Kaplan, A. Klimek, J. Kuklík, K. Novotný und Z. Kufnerová, Z. Zeman und weitere), als mich jedoch selbst mein alter Kommilitone und Fachmann auf diesem Gebiet Milan Hauner nicht ganz entmutigt hatte, trug ich das deutsche Original ${ }^{26}$ im MUMOK vor. Ein ehemaliger Mitarbeiter aus der tschechischen Botschaft in Bonn machte meinen tschechischen Verleger

23 Das Buch erschien jedoch erst am Anfang des Jahres 2011. Das war eine Marketingentscheidung des Verlags, damit der Titel in der Vorweihnachtszeit mit vielen Titeln nicht unterzugeht. Eine Anmerkung dazu ist in den Tagebüchern von Jiří Gruša (im Besitz von Frau Sabine Gruša) zu finden.

24 Obzwar diese Bemerkung über die Beilage in der Werkausgabe bei Wieser Verlag geblieben ist, ist sie in dem Buch nirgends zu finden! In der tschechischen Ausgabe sind zwei Beilagen, diese ist als Beilege No. 1 (Př́loha č. 1) gekennzeichnet.

25 In BAÖ kam es zu einem Irrtum, als der Name des Historikers Bořivoj Čelovský (BJR) auf B. Čelakovský geändert wurde. Ein weiterer Unterschied ist, dass BJR die vollen Taufnamen bringt.

26 Im tschechischen Original heißt es: „sestavil jsem kompletní německou verzi“. 
darauf aufmerksam und wurde zum Übersetzer der Brünner Ausgabe. Für den tschechischen Leser musste man manches ergänzen, was wiederum in dieses Buch einfloss. “27

Dazu noch in der deutschsprachigen Ausgabe im Nachwort von Michael Frank (In hilflosem Trotz - Jiř́ Gruša und der Rechtsnihilismus der tschechischen Gesellschaft) im letzten Absatz:

„Vorträge zum Thema Beneš, das hier ja wurzelt, hatten ein hochintensives Echo, beipflichtend wie empört, auf jeden Fall aber anzeigend, wie sehr besonders die tschechische Gesellschaft von diesem historischen Geist weiter umgetrieben wird. Daraus entstand dieser Band, erst auf Tschechisch dann auf Deutsch." (BAÖ, S. 187)

Äußerlich scheinen die beiden Ausgaben sehr ähnlich, fast identisch zu sein: die Anzahl und Reihenfolge der Kapitel stimmen überein; die einzige kleine Abweichung ist bei der Übersetzung des Kapiteltitels „Přes Moskvu do Prahy“ - „Von Moskau nach Prag“. Bemerkenswert ist, dass auch in der deutschen Ausgabe der Titel des Kapitels „Odsun“ so tschechisch bleibt - das entspricht der Tatsache, wie diese tschechische „mildernde“ Bezeichnung für den Transfer der deutschsprachigen Bevölkerung, die im deutschsprachigen Raum hart als Vertreibung bezeichnet wird, in den gemeinsamen Raum und Verständigung getreten ist.

Die weitere Gestaltung des Buchs im tschechischen Original beinhaltet neben Anmerkungen (in beiden Ausgaben - jedoch teilweise unterschiedlich, zu dem Thema noch später) noch darüber hinaus zwei Beilagen, ${ }^{28}$ Sekundärliteratur und Namensregister. Diese Ausstattung in der Werkausgabe ${ }^{29}$ fehlt.

Beim Textvergleich kommen wir dazu, dass es sich keinesfalls um eine exakte Übersetzung handelt, sondern eher um eine Autorenübertragung mit vielen neuen schöpferischen Erfindungen im Text und Anpassungen an den neuen Rezeptionskreis. Hier einige Beispiele:

„Vůdcův řev vracel do českých uší degradační duktus kakánských zupáků (...) Český posluchač nevnímal obsah, ale akcent a gestikulaci. Ta na něj dělala dojem ochotnictví a brutality v jednom.“ (BJR, S. 10.)

„Führers Gebrülle brachte in das tschechische Ohr den Duktus der kakanischen Obstschnäpsler zurück (...) Der Tscheche nahm nicht den Inhalt wahr, sondern das Gehabe. Der Redner kam ihm vor wie ein Dilettant und Draufgänger." (BAÖ, S. 24)

„A Hudla i jako mladý muž prohloubí svou českou zkušenost. Ač budoucí nadčlověk, žije nejprve jako podnájemník ve čtvrti Mariahilf (U Panny Marie Pomocné), kde se to Čechy a dalšími

27 Im tschechischen Original heißt es: „Ta [gemeint ist die Vorleseung im MUMOK - Anm. MJ] tvoří bázi i tohoto př̀kladu. Zajímal se o ni totiž někdejší spolupracovník na bonnské ambasádě dr. Mojmír Jeřábek a informoval o ní mého brněnského nakladatele. Pro český kontext bylo třeba ledacos doplnit a za podněty v tomto smyslu děkuji M. Jeřábkovi zvlášt vydatně.“

28 Die Beilage No. 1 wurde schon erklärt - vgl. Fußnotiz 17. Die tschechische Beilage No. 2 stammt von Jan Kratochvil (Museum des tschechischen und slowakischen Exils in Brünn). Der Text erweitert das Kapitel Čechoslovakista über den Tod des Generals Milan Rastislav Štefánik und bringt Dokumente mit Äußerungen dazu von E. Beneš und T. G. Masaryk.

29 Beneš als Österreicher erschien als Band Essyas III in der Jiří-Gruša-Werkausgabe (Gesammelte Werke in 10 Bänden) beim Wieser Verlag, Klagenfurt/Celovec 2014. 
přistěhovalci jen hemží. Jemu pomáhá Marie Zákrejs. Pronajala mu za 10 korun $10 \mathrm{~m} 2$ předsklepí v Stumpergasse, „Na Pařezech“ či „na Pahýlech“, a Hudla se tam tak cítí. Štěnice, prach a pach. Ačkoli jeho spolubydlící Kubíček pokládá Marii za Polku, je to Poličanka.“ (BJR, S. 11) „Und Hýdla hat bereits als Wiener Bursche seine Tschechenerfahrung vertieft. Obwohl ein zukünftiger Übermensch, wohnte er als Untermieter bei einer Tschechin. In der Mariahilfer Straße ${ }^{30}$ wo es von Tschechen nur so wimmelte. Ihm hat Marie Zákrejs geholfen. Sie überließ ihm für 10 Kronen 10 Quadratmeter Vorkeller der Stumpergasse und er fühlte sich dort ziemlich stumpf: Läuse, Staub und Stunk. Obwohl Hýdlas Mitbewohner die Zákrejs für eine Polin hielt, irrte er, sie kam aus Polička." (BAÖ, S.11)

$\mathrm{Zu}$ diesen Zitaten sei bemerkt, dass man hier die für den Autor typische, von ihm geliebte und oft benutzte Alliteration findet (Dilettant und Draufgänger, prach a pach, Staub und Stunk) und dass in der tschechischen Ausgabe das Wort kakánských mit einer Anmerkung versehen ist, in der über seinen Ursprung bei Robert Musil die Rede ist.

Weitere Beispiele der Textänderungen:

„jistý Hüber (...) Huber (vlastně Hüber)“ (BJR, S. 15) - „Ein gewisser Huber“ (BAÖ, S. 31) „posléze také hagiograf druhého prezidenta první republiky“ (BJR, S. 16) - „Der Hagiograf des zweiten Präsidenten“ (BAÖ, S. 32)

Im Kapitel Odsun (BAÖ, S. 135) hat der Autor sogar einen ganzen einführenden Absatz dazugeschrieben! An vielen anderen Stellen dieses Kapitels hat er umformuliert, stilistisch geändert oder den Text erweitert oder auch gekürzt. Z. B:

„rakouský flair“ (BJR, S. 90) - „kakanisches Flair“ (BAÖ, S. 135)

„Beneš jako Rakušan měl rakouskou ideu, jak vyřešit z Rakouska přejatý problém.“ (BJR, S. 90) - „Beneš als Österreicher griff hier lunatisch zu.“ (BAÖ, S. 135)

„(...) vyprodukuje 143 (slovy: sto třiačtyřicet) těchto dokumentů - neboli téměř jeden denně.“ (BJR, S. 90) - „(...) produziert er die wichtigsten Dekrete, zusammengerechnet (1940-1945) 143 Stück (...)“ (BAÖ, S. 136)

„Národ je třeba obrodit.! (Fettschtift von JG - mj, BJR, S. 91) - „Die Nation wird naturalisiert.“ (BAÖ, S. 136)

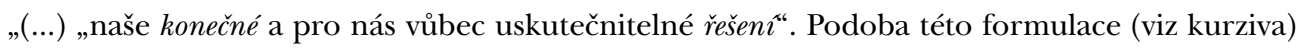
s německým Endlösung mu jistě nedocházela.“ (BJR, S. 91) - „(...) sprach über die „Verbindungen der nationalen Revolution mit der sozialistischen“, die für uns „eine End- und Sachlösung“ überhaupt sein müsse." (BAÖ, S. 32)

„«Spořádaný transfer« bylo navíc nutné teprve zúřadovat (...) takže již v roce 1947 mohl být vyhlášen jeho konec“ (BJR, S. 91) - „Einen „geordneten Transfer“ musste man zudem amtlich verdauen (...) Bereits im Jahre 1946 konnte man das Ende der Aktion verkünden (...)“ (BAÖ, S. 137)

Das Kapitel „... und die Tribunale“

„Jiří Stříbrný (...) jeden z „mužů 28. října“, kteří československou samostatnost vyhlásili doma,

30 Eigentlich geht es um den Wiener Bezirk Mariahilf - vgl. den tschechischen Text oben! 
a tím toto datum proměnili v dodnes platný svátek, dostane doživotí. (...) A přežije ho [Beneše - ergänzt von MJ] o celých sedm let ve valdickém kriminále. Také Rudolf Beran, Benešův politický rival a ministerský předseda po mnichovské krizi (...) musí do Leopoldova“ (BJR, S. 94) - Jiří Stř́ibrný (...) - musste hinter Gitter, obwohl er einer der „Männer des 28. Oktobers“ war, die die tschechoslowakische Selbstständigkeit proklamierten. (...) Er wird ihn um ganze acht Jahre überleben, wenn auch auf einer Pritsche. Auch Rudolf Beran, Ministerpräsident während der Münchner Krise (...) Er saß schon während des Krieges, musste aber auch danach in die Zelle.“ (BAÖ, S. 142)

„Také farář Tiso, vůdce separatistických Slováků“ (BJR, S. 95) - „Und der slowakische Tiso, ein Pfarrer der aus der Pfarrerrepublik“ (BAÖ, S. 142).

Neben diesen Textänderungen aus oben beschriebenen Gründen (sprachlichen, stilistischen, ästhetischen etc.), hat der Autor auch die neue sprachliche Fassung dem neuen Publikum und den Lesern in dem Notizapparat (BJR) und Anmerkungen (BAÖ) angepasst. Was dem tschechischen Leser seiner Meinung nach nicht erklärt werden musste, musste man dem deutschen Leser näher bringen - es ging vor allem um einige Namen und Fakten aus der tschechischen und tschechoslowakischen Geschichte (z. B. Prokop Drtina, Václav Černý, Ladislav Feierabend, Jan Neruda, Karel Čapek, Jaroslav Seifert, Bohumil Hrabal uvm.). Ähnlich war es bei einigen weiteren Fakten - Ortsnamen: Polička mit dem „Wiener Kontext“ und Erwähnung als Geburtsort von Bohuslav Martinů, Geschichtsereignissen: die Mission von Sir Walter Runciman. Umgekehrt schritt der Autor auch zu Verkürzungen der Erklärungen aus BJR z. B. bei der Herkunfts- und Familiengeschichte von Adolf Hitler (BJR, S. 120-121). Er erklärt dem tschechischen Leser auch den Ausdruck „kakánský“ und „Kakánie“ samt der Herkunft bei Robert Musil (BJR, S. 120). In einigen Fällen kam er auch bei der Überprüfung der Fakten zu Daten-Korrekturen (z. B. Anm. No. 6, S. 167, No. 19, S. 168 oder No. 33, S. 169, alles aus BAÖ).

Zusammenfassend: Der Vergleich der beiden Ausgaben vom „Beneš-Buch“ oder „Psychogramm“, wie es Jiří Gruša selber nennt, ${ }^{31}$ hat bewiesen, dass wenn ein mehrsprachiger Autor sich selbst in eine andere seiner literarischen Sprachen übersetzt, handelt es sich meistens nicht um eine Übersetzung im richtigen und korrekten Sinne des Wortes, also um eine möglichst texttreue Übertragung des Textes aus der Sprache A in die Sprache B, sondern um eine viel freiere schöpferische Tätigkeit, die wir vielleicht als eine „Autorenübersetzung“ bezeichnen könnten. Ota Filip, der mit einigen seinen deutschgeschriebenen Romanen Ähnliches erlebt hat, schreibt deswegen in die tschechischen Ausgaben: do češtiny převyprávěno autorem (vom Autor ins Tschechische nachgeschildert). Milan Kundera, der ziemlich treu, seine meistens essayistische Texten ${ }^{32}$ aus dem Französischen ins Tschechische überträgt, schreibt immer von „počeštění“, also von der

31 Siehe Vorwort und das oben zitierte Interview, Anm. 2 und 17

32 Es stimmt zwar, dass er viele seine Essays ins tschechische übertragen hat und zu seinen französisch verfassten Romanen noch nicht gekommen ist, es gibt jedoch kleine Ausnahmen. Siehe ein Kapitel aus seinem neuesten Buch Fest der Bedeutungslosigkeit (La fête de l'insignifiance, Gallimard Paris 2014) Evin strom in der Revue Kontexty, 4/2016, S. 87. 
„Tschechisierung“ seiner Texte. Jedoch auch bei ihm - oder gerade bei ihm! - kann man den wesentlichen Unterschied zwischen seiner sprachlichen und literarischen Meisterleistung - und den anderen Versuchen um die Übersetzung seiner Texte, die im Internet häufig zu finden (und illegal) sind, weil das der Autor verboten hat. Wohl bestärkt durch die Erfahrung, die ich mich bemüht habe, in diesem Text zu beschreiben.

PhDr. Mojmír Jeřábek / jerabek@czech.cz

Tschechisches Zentrum Wien, Herrengasse 17, A-1010 Wien, AT 